\title{
Tumor Markers in Clinical Practice: A Review Focusing on Common Solid Cancers
}

\author{
Michael J. Duffy \\ Department of Pathology and Laboratory Medicine, St Vincent's University Hospital, Dublin, Ireland
}

\section{Key Words}

Tumor markers $\cdot$ Biomarkers $\cdot \alpha$-Fetoprotein $\cdot$ Human chorionic gonadotrophin - Carcinoembryonic antigen •

Prostate-specific antigen - CA 15-3 • CA 125

\begin{abstract}
Tumor markers are playing an increasingly important role in cancer detection and management. These laboratory-based tests are potentially useful in screening for early malignancy, aiding cancer diagnosis, determining prognosis, surveillance following curative surgery for cancer, up front predicting drug response or resistance, and monitoring therapy in advanced disease. Clinically useful markers include fecal occult blood testing in screening for early colorectal cancer, carcinoembryonic antigen in the management of patients with colorectal cancer, both $\alpha$-fetoprotein and human chorionic gonadotrophin in the management of patients with nonseminomatous germ cell tumors, CA 125 for monitoring therapy in patients with ovarian cancer, estrogen receptors for predicting response to hormone therapy in breast cancer, human epidermal growth factor receptor 2 for the identification of women with breast cancer likely to respond to trastuzumab (Herceptin) and KRAS mutational status for identifying patients with advanced colorectal cancer likely to benefit from treatment with the anti-epidermal growth
\end{abstract}

factor receptor antibodies, cetuximab and panitumumab. Although widely used, the value of prostate-specific antigen screening in reducing mortality from prostate cancer is unclear.

Copyright @ 2012 S. Karger AG, Basel

\section{Introduction}

Tumor markers may be defined as molecules which indicate the presence of cancer or provide information about the likely future behavior of a cancer (i.e., likelihood of progression or response to therapy) $[1,2]$. In asymptomatic subjects, tumor markers are potentially used in screening for early malignancy. In symptomatic patients, markers may help in the differential diagnosis of benign and malignant disease. Following diagnosis and surgical removal of a malignancy, markers may be used for assessing prognosis, postoperative surveillance, therapy prediction and monitoring response to systemic therapy $[1,2]$.

Irrespective of its application, an ideal tumor marker should exhibit the following characteristics:

- possess a high positive and negative predictive value;

- have an inexpensive, simple, standardized and automated assay with clearly defined reference limits; 
Table 1. Biomarkers that have undergone or are currently undergoing evaluation in screening asymptomatic subjects for cancer

\begin{tabular}{ll}
\hline Marker or test & Malignancy \\
\hline FOBT & colorectal \\
PSA & prostate \\
CA 125 & ovarian \\
VMA/HVA & neuroblastoma \\
AFP & hepatocellular \\
Pepsinogen & gastric \\
HCG & trophoblastic \\
\hline
\end{tabular}

VMA = Vanillylmandelic acid; HVA = homovanillic acid.

${ }^{1}$ Only in high-risk areas/high-risk subjects.

${ }^{2}$ In patients who have had a previous hydatidiform mole.

- be acceptable to subjects undergoing the test; and

- have its clinical value validated in a large prospective trial.

Suffice it to state, the ideal tumor marker does not currently exist. Despite this, several markers are now indispensable in the management of patients with cancer. The aims of this article are to review the most widely measured markers in clinical practice and summarize published guidelines for their use.

\section{Use of Markers in Screening for Cancer}

Screening involves the detection of early disease or a preclinical state in subjects without signs or symptoms of disease. Unlike disease diagnosis, screening is performed on individuals without any clinical sign of disease. Compared to established screening tests such as mammography for breast cancer, the Papanicolaou test for cervical cancer and colonoscopy for colorectal cancer (CRC), the use of tumor markers might be expected to have practical advantages in cancer screening [3]. These advantages include [3]:

- Markers can be measured in fluids such as blood and urine that can be obtained with minimal inconvenience to the individuals undergoing screening.

- For many markers, automated assays are available, allowing the processing of large numbers of samples in a relatively short period of time.

- Tests for markers provide quantitative results with objective endpoints.

- Assays for markers are relatively cheap compared to radiological, histological and endoscopy procedures.
Despite these advantages, markers have several limitations as cancer screening tests. In particular, lack of sensitivity for early invasive disease or premalignant lesions and lack of specificity for malignancy limit the use of existing markers in screening asymptomatic subjects for early malignancy $[1,3]$. The dual problem of limited sensitivity and specificity, especially when combined with the low prevalence of most cancers in the community, means that markers, if used alone, have low positive predictive values in screening asymptomatic populations. Indeed, it is the low prevalence of cancer in the general population that prohibits most biomarkers from being used in screening for cancer $[1,3]$. Despite this, a number of markers have either undergone or are presently undergoing evaluation for cancer screening (table 1). Some of the most widely investigated markers in cancer screening are now discussed.

\section{Fecal Occult Blood Testing in Screening for CRC}

One of the best validated screening markers is the use of fecal occult blood testing (FOBT) in screening for CRC. Indeed, 4 large randomized prospective trials carried out in Europe and North America have all shown that screening apparently healthy subjects over 50 years of age using FOBT significantly reduced mortality from CRC [4]. Combined results from the 4 trials showed that the screening resulted in a $16 \%$ reduction in the relative risk of CRC mortality [4]. Following adjustment for those subjects that failed to attend screening, mortality reduction increased to $25 \%$.

Screening healthy individuals aged $\geq 50$ years using FOBT is now recommended, especially in Europe [5, 6]. Although the 4 prospective randomized trials mentioned above all used a guaiac-based FOBT, both the European Group of Tumor Markers (EGTM) and a European Union expert panel currently recommend the use of a fecal immunochemical test in screening for CRC $[5,6]$. As previously reviewed, the use of fecal immunochemical tests have several advantages vis-à-vis FOBTs, including greater analytical sensitivity and specificity, improved clinical performance and potential for automation [5].

\section{Prostate-Specific Antigen in Screening for Prostate Cancer}

In contrast to FOBT in screening for CRC, the clinical value of prostate-specific antigen (PSA) in screening for prostate cancer is less clear [7]. In 2009, results from 2 large randomized prospective trials evaluating the screening potential of this marker were published. The first of these trials was carried out in the USA and involved 76,693 men that were randomized to either an- 
nual screening or regular care [8]. Following analysis at 7-10 years of follow-up, death rates were similar in the 2 groups. A limitation of this study was that approximately $50 \%$ of men in the control group underwent screening during the study. This trial could thus be regarded as a comparison between a heavily screened group and a less heavily screened group rather than a true randomized trial [8]. A further limitation was that approximately $40 \%$ of the men participating had a PSA test prior to the start of the trial. These men were thus less likely to be diagnosed with prostate cancer during the trial proper.

In a somewhat similar but larger trial carried out in Europe, 162,243 men were randomly allocated to PSA screening or to a control group not subjected to screening [9]. After a median follow-up of 9 years, death rates from prostate cancer were found to be $20 \%$ lower in the screened compared to the control group. This apparent benefit, however, resulted in major overdetection and overtreatment. Thus, according to the paper's authors, 1,410 men would have to be screened and 48 additional cases of prostate cancer would have to undergo treatment to prevent 1 death from prostate cancer [9].

With these conflicting findings it is not surprising that guidelines published by expert panels vary with respect to PSA screening for prostate cancer, i.e., while some groups recommend screening, others are opposed to the practice $[7,10]$. Although expert panels disagree on whether or not to recommend PSA screening, most recommend that prior to undergoing screening for prostate cancer, men should be informed of the risks and benefits of early disease detection [7]. Only after receiving such information should PSA screening be initiated.

\section{CA 125 in Screening for Ovarian Cancer}

For the past decade or so, 2 large prospective randomized trials have been evaluating the role of CA 125 and transvaginal ultrasound in screening for ovarian cancer $[11,12]$. One of these trials recently reported its findings [13]. In this trial carried out in the USA, 78,216 women aged 55-74 years were randomized to undergo either annual screening with CA 125 for 6 years and transvaginal ultrasound for 4 years or standard care. After 13 years of follow-up, death rates were similar in the screened and control groups. Clearly, in this trial, screening with CA 125 and transvaginal ultrasound did not reduce ovarian cancer mortality [13]. According to the EGTM guidelines, CA 125 either alone or in combination with other modalities cannot be recommended in screening for ovarian cancer in asymptomatic women outside the context of a randomized controlled trial [14].

\section{$\alpha$-Fetoprotein in Hepatocellular Cancer}

Subjects at increased risk of developing hepatocellular cancer (HCC) include those with cirrhosis due to infection with hepatitis $B$ virus or hepatitis $C$ virus, genetic hemochromatosis or biliary cirrhosis. Guidelines published by expert panels differ in their recommendations regarding the use of $\alpha$-fetoprotein (AFP) in screening for HCC. Thus, the National Academy of Clinical Biochemistry USA (NACB) [15] state that, 'AFP should be measured and abdominal ultrasound performed at sixmonthly intervals in patients at high risk of HCC, especially in those with hepatitis B and hepatitis C-related liver cirrhosis. AFP concentrations that are $>20 \mu \mathrm{g} / \mathrm{l}$ and showing consistent increases in concentration should prompt further investigation even if ultrasound is negative.' On the other hand, the American Association for the Study of Liver Disease recommend that surveillance of high-risk subjects should be performed only with ultrasound. According to this organization, AFP should only be used when ultrasound is not available. This latter recommendation is based on the limited use of AFP in detecting early HCC.

\section{Use of Markers as Diagnostic Aids for Cancer}

As discussed above with screening, limited sensitivity for small or early cancers and lack of tumor specificity preclude the use of serum markers for the primary diagnosis of cancer. In a limited number of situations, however, markers may aid in the differential of benign and malignant disease. Thus, CA 125 is used as an adjunct in differentiating between benign and malignant pelvic masses in postmenopausal women [14]. The EGTM recommend that CA 125 should be measured in postmenopausal women presenting with such masses. According to this expert panel, patients with elevated levels (e.g. $>35$ U/l) should be considered for referral to a surgeon specialized in gynecological oncology surgery [14]. This recommendation was based on studies showing superior outcome in patients with ovarian cancer if treated by a gynecological oncologist rather than by a general surgeon [16].

Another marker that may be helpful in cancer diagnosis is AFP in the detection of HCC. According to the American Association for the Study of Liver Disease, finding a hepatic mass of $>2 \mathrm{~cm}$ in diameter in a patient with a cirrhotic liver is highly suspicious of HCC. If AFP is $>200 \mu \mathrm{g} / \mathrm{l}$ and the radiological appearance is suggestive of HCC, the likelihood is that the lesion is HCC and biopsy is not essential [17]. Similar recommendations have 
Table 2. Markers that may be used for determining prognosis in different cancers

\begin{tabular}{ll}
\hline Cancer & Marker(s) \\
\hline Breast & Oncotype DX, uPA/PAI-1 \\
Germ cell & AFP, HCG, LDH \\
Colorectal & CEA, MSI \\
Prostate & PSA \\
Ovarian & CA 125 \\
\hline
\end{tabular}
bility.

LDH $=$ Lactate dehydrogenase; MSI $=$ microsatellite insta -

been published by the NACB [15]. AFP, however, is of limited value in aiding the diagnosis of lesions $<2 \mathrm{~cm}$ in diameter.

\section{Use of Markers in Assessing Prognosis}

Prognostic markers provide information on the likely outcome following diagnosis of a disease. Such markers may help avoid undertreatment of patients with aggressive disease and overtreatment of those with indolent disease. Prognostic markers are most important in cancers that vary widely in their outcome such as prostate and breast cancer. In these cancers, prognostic markers may help identify those patients with aggressive disease that could benefit from additional therapies and simultaneously select those patients who may not require additional therapy.

Prognostic markers are most widely used in breast cancer, especially in the subset with lymph node-negative disease. In this subgroup of patients with breast cancer, prognostic markers help identify patients who may be spared the toxicity and side effects of adjuvant chemotherapy. The 2 best validated prognostic markers for breast cancer are the Oncotype DX test and uPA/PAI1. The Oncotype DX test measures the expression of 16 cancer-associated and 5 control genes by RT-PCR on RNA isolated from paraffin-embedded breast cancer tissue [18]. This test is currently available from a commercial laboratory in the USA. Although the Oncotype DX test is recommended by several expert panels including the American Society of Clinical Oncology (ASCO), NACB and EGTM $[10,19,20]$, the test has not yet been validated in a large prospective randomized trial. Such a study, however, is ongoing as part of the TAILORx trial.

Several Tumor Markers in Clinical Practice
uPA and PAI-1 are the best validated prognostic markers in breast cancer, especially for patients with lymph node-negative disease [21]. uPA is a protease that mediates invasion and metastasis. Although PAI-1 normally functions as an endogenous inhibitor of uPA activity, at the high concentrations frequently present in tumors it appears, like uPA, to also play a role in cancer spread [21]. Unlike any other marker in breast cancer, the prognostic impact of UPA and PAI-1 has been validated in both a multicenter randomized prospective trial and a pooled analysis of raw data from several small retrospective and a prospective study $[22,23]$, i.e., in 2 level I evidence studies.

As with Oncotype DX, measurement of uPA/PAI-1 is recommended by a number of expert panels $[10,19,20]$. According to ASCO, 'uPA and PAI-1 may be used for the determination of prognosis in patients with newly diagnosed, node negative breast cancer. Low levels of both markers are associated with a sufficiently low risk of recurrence, especially in hormone receptor-positive women who will receive adjuvant endocrine therapy, that chemotherapy will only contribute minimal additional benefit. Furthermore, CMF-based adjuvant chemotherapy provides substantial benefit, compared with observation alone, in patients with high risk of recurrence as determined by high levels of uPA and PAI-1' [19]. Other widely studied cancer prognostic markers are listed in table 2.

\section{Therapy-Predictive Markers in Cancer}

As mentioned above, therapy-predictive markers are factors that prospectively identify likely response or resistance to a specific treatment. Predictive markers are important in cancer patient management as patients with the same histological type of malignancy respond very differently to a specific drug. Thus, response rates for patients with different types of advanced cancer to currently available systemic treatments vary from about 10 to $>90 \%$ [24]. Many of the newer biological therapies, in particular, have efficacy in only a minority of patients. This finding, when combined with the high costs of these drugs [25], illustrates the importance of having accurate predictive markers.

As well as assessing efficacy, predictive markers may also be able to identify optimum drug dose and predict toxicity. Thus, measurement of predictive markers can increase drug efficacy and result in decreased toxicity. This, in turn, should reduce overall health care costs and lead to an enhanced quality of life for patients [26].

Med Princ Pract 2013;22:4-11 
As with prognostic markers, predictive markers are most developed for breast cancer. Thus, in breast cancer, measurement of estrogen receptors and progesterone receptors is universally used to identify patients for treatment with hormone therapy (tamoxifen or aromatase inhibitor), while the assay of human epidermal growth factor receptor 2 is routinely used to select patients for treatment with trastuzumab (Herceptin) or lapatinib [27]. Newly introduced predictive markers include mutant KRAS status for identifying responsiveness to antiepidermal growth factor receptor antibodies (cetuximab and panitumumab) in advanced CRC and epidermal growth factor receptor mutation status for selecting patients with advanced non-small cell lung cancer for treatment with anti-epidermal growth factor receptor kinases (gefitinib and erlotinib) [27].

\section{Use of Markers in Surveillance following Initial Treatment for Cancer}

One of the most frequent uses of tumor markers at present is in the postoperative follow-up of patients following a diagnosis of malignancy (table 3). In several situations, serial levels of cancer markers can predict the presence of early recurrent/metastatic disease, i.e., provide a lead time over clinical or radiological findings. It is assumed that the early detection of recurrent/metastatic disease followed by the initiation of treatment enhances the chance of cure or results in an improved survival [2]. For some cancer types, however, the evidence currently available does not support this widely held assumption. Indeed, the value of makers in postoperative surveillance may vary from cancer to cancer. Below, I discuss the usefulness of markers in surveillance following the diagnosis of different cancers.

\section{Carcinoembryonic Antigen in CRC}

Multiple studies have shown that following curative surgery for CRC, patients undergoing an intensive surveillance regime that included regular carcinoembryonic antigen (CEA) measurements had a significantly better outcome than those undergoing surveillance without CEA testing $[28,29]$. Most expert panels in Europe and the USA therefore recommend serial measurements of CEA following curative surgery for CRC [10, 30-32]. According to the EGTM, 'CRC patients with stage II and III (Dukes' B and C) CRC that may be candidates for either liver resection or systemic treatment in the event of recurrence in that organ, should have CEA measured every 2
Table 3. Serum markers that may be used in postoperative surveillance and monitoring therapy in different cancers

\begin{tabular}{ll}
\hline Cancer & Marker(s) \\
\hline Colorectal & CEA \\
Hepatocellular & AFP \\
Pancreatic & CA 19-9 \\
Ovarian & CA 125 \\
Breast & CA 15-3 \\
Prostate & PSA \\
Germ cell & AFP, HCG \\
Lung (non-small cell) & CYFRA 21-1, SCC \\
Lung (small cell) & NSE, proGRP \\
Melanoma & S100 \\
Trophoblastic & HCG \\
Thyroid (differentiated) & thyroglobulin \\
\hline
\end{tabular}
lase.

SCC $=$ Squamous cell carcinoma; NSE = neuron-specific eno-

to 3 months for at least 3 years after diagnosis' [30, 31]. Although serial measurements of CEA are widely recommended as part of a surveillance regime, agreement is lacking as to the extent of concentration change that constitutes a clinically significant increase in marker levels. According to the EGTM group [30], 'a significant increase in CEA levels occurs if the elevation is at least $30 \%$ over that of the previous concentration'. This organization also states that prior to initiating therapy, any increase must be confirmed by a second sample taken within approximately 1 month. If the second sample is also increased, the patient should undergo further investigations such as imaging [30].

\section{AFP and Human Chorionic Gonadotrophin in}

\section{Patients with Germ Cell Tumors}

Two main histological types of germ cell tumors exist, seminoma and non-seminoma. The use of AFP and human chorionic gonadotrophin (HCG) in monitoring patients with the non-seminomatous type germ cell tumors of the testis is often regarded as approximating the ideal use of tumor markers [2]. This is because these 2 markers are sensitive indicators of germ cell disease status, i.e., whether disease is stable, progressing or regressing. A further reason why these markers are particularly helpful in patients with germ cell tumors is that these malignancies are highly chemosensitive. Indeed, it is now widely accepted that following orchidectomy for non-seminomatous testicular germ cell tumors, increasing AFP or 
HCG levels in the absence of radiological or clinical evidence of disease suggests active disease and may provide sufficient reassurance to initiate treatment, provided likely causes of false-positive marker levels can be eliminated [2].

According to the ASCO guidelines, AFP and HCG should be assayed during surveillance following definite therapy for non-seminomatous germ cell tumors, regardless of stage [33]. These measurements may be carried out every 1-2 months in the first year, every 2-4 months in the second year, every 3-6 months in the third and fourth years, every 6 months in the fifth year and annually thereafter. Surveillance should be continued for at least 10 years after therapy is completed. For monitoring patients with pure seminoma, HCG and/or LDH are generally recommended [33].

\section{CA 125 in Patients with Ovarian Cancer}

The clinical value of serial determination of CA 125 in post-therapy monitoring of patients with a history of ovarian cancer is unclear. Although regular measurement of the marker may detect early recurrences with a median lead time of 4-5 months [34], a recently completed prospective randomized trial found no survival benefit from starting early treatment based on a rising serum CA 125 level [35]. This trial involved 1,442 women previously diagnosed with ovarian cancer but in clinical remission. CA 125 levels were measured every 3 months, but the results were not made available to patients or their doctors. The women were randomized when their marker concentrations reached twice the upper limit of the normal range, to receive treatment immediately or to continue with blinded CA 125 determinations. In this latter situation, women underwent treatment only when there was clinical evidence of recurrence. Despite the earlier introduction of second-line chemotherapy, no significant difference in overall survival was found in the 2 groups. This negative finding may relate, at least in part, to the lack of effective therapy for recurrent ovarian cancer.

Uncertainties therefore exist with respect to the value of CA 125 measurement and timing of treatment for relapsed ovarian cancer. Although the NACB panel currently recommends serial measurement of CA 125 following surgery and initial systemic therapy for ovarian cancer [10], the EGTM panel is opposed to this practice [14]. With such conflicting recommendations, a practical way forward may be to take the patients' wishes into consideration.

Several Tumor Markers in Clinical Practice

\section{PSA in Prostate Cancer}

Irrespective of whether men with diagnosed prostate cancer undergo active treatment (e.g. with radical prostatectomy, radiotherapy or brachytherapy) or active surveillance, regular monitoring with PSA is now commonly carried out [36]. Following successful radical prostatectomy, PSA levels should decline to undetectable levels. A subsequent increase to $\geq 0.2 \mu \mathrm{g} / \mathrm{l}$ is defined as biochemical recurrence [37]. Generally, decreases in PSA levels following radiation therapy are less than those following radical prostatectomy. A rise in PSA levels of $\geq 2 \mu \mathrm{g} / 1$ over and above the nadir value has been proposed as a definition of radiation therapy failure [38]. It is still unclear whether the introduction of salvage therapy based on these definitions of PSA recurrence enhances patient outcome or quality of life.

\section{CA 15-3 in Breast Cancer}

The most widely used marker in surveillance following a diagnosis of breast cancer is CA 15-3 [39]. Although widely used in some countries for this purpose, it is unclear whether serial measurement improves patient outcome. Consequently, guidelines vary with respect to their recommendations on the use of CA 15-3 in monitoring asymptomatic women following a diagnosis of breast cancer. While expert panels such as ASCO recommend against routing use of CA 15-3 in surveillance in the surveillance of breast cancer patients [19], EGTM endorses its measurement in this setting [20]. According to the NACB guidelines [10], 'CA 15-3 should not be routinely used for the early detection of recurrences/metastases in patients with diagnosed breast cancer. However, as some patients, as well as some doctors, may wish to have these measurements, the ultimate decision on whether or not to use CA 15-3 must be taken by the doctor in consultation with the patient.'

\section{Monitoring Systemic Therapy}

Another frequent use of tumor markers is in monitoring patients with advanced cancer receiving systemic therapy (table 3) $[1,2,10]$. The markers used in monitoring therapy in a specific malignancy are the same as those measured in postoperative surveillance (see above). Generally, decreasing levels of markers following the initiation of therapy correlates with tumor regression and increasing levels predict progressive disease. Tumor markers, however, should not be used alone in assessing response to therapy.

Med Princ Pract 2013;22:4-11 
A caveat in the use of markers in monitoring therapy in patients with advanced malignancy is the possible occurrence of transient increases or spikes within the first few weeks of administering therapy. These transient increases appear to be due to tumor cell necrosis or apoptosis in response to the initial treatment with chemotherapy. Such transient increases have not yet been reported with biological therapies such as therapeutic antibodies (e.g. Herceptin, cetuximab or panitumumab).

\section{Conclusion}

From above, it is clear that certain tumor markers are mandatory in the management of patients with certain types of cancer. Indeed, in some situations, markers can be used as the sole criterion for clinical decision making. This applies particularly for therapy-predictive markers such as estrogen receptor and human epidermal growth factor receptor 2 in breast cancer. Another good example is the use of HCG and AFP in therapy decision making in patients with diagnosed non-seminomatous germ cell tumors. In other situations, however, the value of markers is less clear, for example, the role of PSA in screening for prostate cancer, assay of CA 125 following surgery and chemotherapy for ovarian cancer and assay of CA 15-3 in the surveillance of patients following the diagnosis of breast cancer. Hopefully, future studies will provide definite answers on the use of these markers, in the near future. New procedures for measuring tumor markers in the future are likely to focus on gene expression microarray, proteomics and detection of circulating tumor cells.

\section{Acknowledgements}

The authors wish to thank Science Foundation Ireland, Strategic Research Cluster Award (08/SRC/B1410) to Molecular Therapeutics for Cancer Ireland.

\section{References}

1 Duffy MJ: Clinical uses of tumor markers: a critical review. Crit Rev Clin Lab Sci 2001;38: 225-262.

2 Duffy MJ: Role of tumor markers in patients with solid tumors: a critical review. Eur J Int Med 2007;18:175-184.

3 Duffy MJ: Use of biomarkers in screening for cancer. EJIFCC 2010;21, No 1.

4 Hewitson P, Glasziou P, Irwig L Towler B, Watson E: Screening for colorectal cancer using fecal the occult blood test, Hemoccult. Cochrane Database of Syst Rev 2007;1: CD001216.

5 Duffy MJ, van Rossum LG, van Turenhout ST, Malminiemi O, Sturgeon C, Lamerz R, Nicolini A, Haglund C, Holubec L, Fraser CG, Halloran SP: Use of faecal markers in screening for colorectal neoplasia: a European group on tumor markers position paper. Int J Cancer 2011;128:3-11.

6 Segnan N, Patnik J, von Karsa L (eds): European Guidelines for Quality Assurance in Colorectal Cancer Screening and Diagnosis. Luxembourg, Publication Office of the European Union, 2010.

7 Duffy M: Prostate-specific antigen: does the current evidence support its use in prostate cancer screening? Ann Clin Biochem 2011; 22:2234-2240.

8 Andriole GL, Crawford ED, Grubb RL 3rd, Buys SS, Chia D, Church TR, Fouad MN, Gelmann EP, Kvale PA, Reding DJ, et al: Mortality results from a randomized prostate-cancer screening trial. N Engl J Med 2009;360:1310-1319.
Schröder FH, Hugosson J, Roobol MJ, Tammela TL, Ciatto S, Nelen V, Kwiatkowski M, Lujan M, Lilja H, Zappa M, et al: Screening and prostate-cancer mortality in a randomized European study. N Engl J Med 2009;360: $1320-1328$

10 Sturgeon CM, Duffy MJ, Stenman UH, Lilja $\mathrm{H}$, Brünner N, Chan DW, Babaian R, Bast RC Jr, Dowell B, Esteva FJ, National Academy of Clinical Biochemistry, et al: National Academy of Clinical Biochemistry laboratory medicine practice guidelines for use of tumor markers in testicular, prostate, colorectal, breast and ovarian cancers. Clin Chem 2008;54:e11-e79.

11 Buys SS, Partridge E, Greene MH, Prorok PC, Reding D, Riley TL, Hartge P, Fagerstrom RM, Ragard LR, Chia D, et al: Ovarian cancer screening in the Prostate, Lung, Colorectal and Ovarian (PLCO) cancer screening trial. Am J Obstet Gynecol 2005; 193:1630-1639.

12 Menon U, Gentry-Maharaj A, Hallett R, Ryan A, Burnell M, Sharma A, Lewis S, Davies S, Philpott S, Lopes A, et al: Sensitivity and specificity of multimodal and ultrasound screening for ovarian cancer, and stage distribution of detected cancers: results of the prevalence screen of the UK Collaborative Trial of Ovarian Cancer Screening (UKCTOCS). Lancet Oncol 2009;10:327340.
Buys SS, Partridge E, Black A, Johnson CC, Lamerato L, Isaacs C, Reding DJ, Greenlee RT, Yokochi LA, Kessel B, PLCO Project Team, et al: Effect of screening on ovarian cancer mortality: the Prostate, Lung, Colorectal and Ovarian (PLCO) Cancer Screening Randomized Controlled Trial. JAMA 2011;305:2295-2303.

14 Duffy MJ, Bonfrer JM, Kulpa J, Rustin GJ, Soletormos G, Torre GC, Tuxen MK, Zwirner M: CA125 in ovarian cancer: European Group on Tumor Markers (EGTM) guidelines for clinical use. Int J Gynecol Cancer 2005; 15:679-691.

- 15 Sturgeon CM, Duffy MJ, Hofmann BR, Lamerz R, Fritsche HA, Gaarenstroom K, Bonfrer J, Ecke TH, Grossman HB, Hayes P, National Academy of Clinical Biochemistry, et al: National Academy of Clinical Biochemistry Laboratory Medicine Practice Guidelines for use of tumor markers in liver, bladder, cervical, and gastric cancers. Clin Chem 2010;56:e1-e48.

16 Earle CC, Schrag D, Neville BA, Yabroff KR, Topor M, Fahey A, Trimble EL, Bodurka DC, Bristow RE, Carney M, Warren JL: Effect of surgeon specialty on processes of care and outcomes for ovarian cancer patients. J Natl Cancer Inst 2006;98:172-180.

17 Bruix J, Sherman M: Management of hepatocellular carcinoma. Hepatology 2005;42: $1208-1236$ 
18 Paik S, Shak S, Tang G, Kim C, Baker J, Cronin M, Baehner FL, Walker MG, Watson D, Park T, et al: A multi-gene assay to predict recurrence of tamoxifen-treated node-negative breast cancer. N Engl J Med 2004;351: 2817-2826.

19 Harris L, Fritsche H, Mennel R, Norton L, Ravdin P, Taube S, Somerfield MR, Hayes DF, Bast RC Jr, American Society of Clinical Oncology: American Society of Clinical Oncology 2007 update of recommendations for the use of tumor markers in breast cancer. J Clin Oncol 2007;25:5287-5312.

20 Molina R, Barak V, van Dalen A, Duffy MJ, Einarsson R, Gion M, Goike H, Lamerz R, Nap M, Sölétormos G, Stieber P: Tumor markers in breast cancer - European Group on Tumor Markers recommendations. Tumour Biol 2005;26:281-293.

21 Duffy MJ: Urokinase plasminogen activator and its inhibitor, PAI-1, as prognostic markers in breast cancer: from pilot to level 1 evidence. Clin Chem 2002;48:1194-1197.

22 Janicke F, Prechtl A, Thomssen C, Harbeck $\mathrm{N}$, Meisner C, Untch M, Sweep CG, Selbmann HK, Graeff H, Schmitt M, German N0 Study Group: Randomized adjuvant chemotherapy trial in high-risk node-negative breast cancer patients identified by urokinase-type plasminogen activator and plasminogen activator inhibitor type 1 . J Natl Cancer Instit 2001;93:913-920.

-23 Look MP, van Putten WL, Duffy MJ, Harbeck N, Christensen IJ, Thomssen C, Kates R, Spyratos F, Fernö M, Eppenberger-Castori S, et al: Pooled analysis of prognostic impact of tumor biological factors uPA and PAI-1 in 8,377 breast cancer patients. J Natl Cancer Instit 2002;94:116-128.

24 Duffy MJ: Predictive markers in breast and other cancers: a review. Clin Chem 2005;51: 494-503.
25 Fojo T, Parkinson DR: Biologically targeted cancer therapy and marginal benefits: are we making too much of too little or are we achieving too little by giving too much? Clin Cancer Res 2010;16:5972-5980.

26 Huang RS, Ratain MJ: Pharmacogenetics and pharmacogenomics of anticancer agents. CA Cancer J Clin 2009;59:42-55.

27 Duffy MJ, O’Donovan N, Crown J: Use of molecular markers for predicting therapy response in cancer patients. Cancer Treat Rev 2011;37:151-159.

$\checkmark 28$ Renehan AG, Egger M, Saunders MP, O'D wyer ST: Impact on survival of intensive follow-up after curative resection for colorectal cancer: systematic review and meta-analysis of randomised trials. BMJ 2002;324:813-820.

-29 Figueredo A, Rumble RB, Maroun J, Earle CC, Cummings B, McLeod R, Zuraw L, Zwaal C, Gastrointestinal Cancer Disease Site Group of Cancer Care Ontario's Program in Evidence-based Care: Follow-up of patients with curatively resected colorectal cancer: a practice guideline. BMC Cancer 2003;3:26-38.

30 Duffy MJ, van Dalen A, Haglund C, Hansson L, Klapdor R, Lamerz R, Nilsson O, Sturgeon C, Topolcan O: Clinical utility of biochemical markers in colorectal cancer: European Group on Tumour Markers (EGTM) guidelines. Eur J Cancer 2003;39: 718-727.

31 Duffy MJ, van Dalen A, Haglund C, Hansson L, Holinski-Feder E, Klapdor R, Lamerz R, Peltomaki P, Sturgeon C, Topolcan O: Tumour markers in colorectal cancer: European Group on Tumour Markers (EGTM) guidelines for clinical use. Eur J Cancer 2007; 43:1348-1360.

32 Locker GY, Hamilton S, Harris J, Jessup JM, Kemeny N, Macdonald JS, Somerfield MR, Hayes DF, Bast RC Jr, ASCO: ASCO 2006 update of recommendations for the use of tumor markers in gastrointestinal cancer. J Clin Oncol 2006;24:5313-5327.
Gilligan TD, Seidenfeld J, Basch EM, Einhorn LH, Fancher T, Smith DC, Stephenson AJ, Vaughn DJ, Cosby R, Hayes DF, American Society of Clinical Oncology: American Society of Clinical Oncology Clinical Practice Guideline on uses of serum tumor markers in adult males with germ cell tumors. J Clin Oncol 2010;28:3388-3404.

34 Tuxen MK, Soletormos G, Dombernowsky P: Serum tumour marker CA 125 in monitoring of ovarian cancer during first-line chemotherapy. Br J Cancer 2001;84:13011307.

35 Rustin GJ, van der Burg ME, Griffin CL, Guthrie D, Lamont A, Jayson GC, Kristensen G, Mediola C, Coens C, Qian W, et al, MRC OV05; EORTC 55955 investigator: Early versus delayed treatment of relapsed ovarian cancer (MRC OV05/EORTC 55955): a randomised trial. Lancet 2010;376:1155-1163.

36 Hernandez J, Thompson IM: Prostate-specific antigen: a review of the validation of the most commonly used cancer biomarker. Cancer 2004;101:894-904.

37 Cookson MS, Aus G, Burnett AL, CanbyHagino ED, D'Amico AV, Dmochowski RR, Eton DT, Forman JD, Goldenberg SL, Hernandez J, et al: Variation in the definition of biochemical recurrence in patients treated for localized prostate cancer: the American Urological Association Prostate Guidelines for Localized Prostate Cancer Update Panel report and recommendations for a standard in the reporting of surgical outcomes. J Urol 2007;177:540-545.

38 Horwitz EM, Thames HD, Kuban DA, Levy LB, Kupelian PA, Martinez AA, Michalski JM, Pisansky TM, Sandler HM, Shipley WU, et al: Definitions of biochemical failure that best predict clinical failure in patients with prostate cancer treated with external beam radiation alone: a multi-institutional pooled analysis. J Urol 2005;173:797-802.

39 Duffy MJ, Evoy D, McDermott EW: CA 15-3: uses and limitation as a biomarker for breast cancer. Clin Chim Acta 2010;411:1869-1874. 